\title{
Relato de experiência em processo de educação permanente: Construção de cartilha participativa sobre cuidados paliativos
}

\author{
Experience report in the continuing education process: Construction of a participatory booklet on
} palliative care

Informe de experiencia en el proceso de educación continua: Construcción de un folleto participativo sobre cuidados paliativos

\section{Resumo}

Atualmente observamos um aumento na cronicidade de doenças não transmissíveis, elevando a estimativa de vida populacional e mostrando a existência do processo de adoecimento que leva a finitude da vida. Automaticamente surge a necessidade de estabelecer um processo de assistência e educação aos Cuidados Paliativos, simbolizando o a aprimoramento do sistema Morte e Morrer. Objetivo: Sabendo que a informação compreende elemento fundamental no processo do desenvolvimento da Educação Permanente na Saúde para o trabalho na assistência ao paciente, retratase a experiência na construção de uma cartilha educativa a respeito de Cuidados Paliativos em um hospital de emergência da Capital Fluminense como um vetor de conhecimento. Metodologia: Foi realizada uma pesquisa descritiva acerca da necessidade de conhecimento pelos profissionais de enfermagem no tema Cuidados Paliativos tornando-se o disparador para a construção desta cartilha. Resultados e discussão: Após a avaliação da aplicação do material foi possível percebê-lo como material agregador do conhecimento interdisciplinar numa equipe multiprofissional.

Palavras-chave: Cuidados paliativos; Paciente oncológico; Qualidade de vida; Materiais educativos e de divulgação; Educação permanente.

\begin{abstract}
Currently, we observe na increase in the chronicity of non-communicable diseases, raising the estimate of population life and showing the existence of the disease process that leads to finitude life. Automatically arises the need to establish a processo $\mathrm{f}$ assistance and education to Palliative Care, symbolizing the improvement of the Death and Dying system. Objective: Knowing that information comprises a fundamental elemento in the processo of developing Permanent Health Education for work in patient care, the exeperience in the construction of an educational booklet about Palliative Care in na emergency hospital in the city of Rio de Janeiro is portrayed as a knowledge vector. Methodology: A descriptive research was carried out on the need for knowledge by nursing professionals on the topic of Palliative Care, which became the trigger for the construction of this booklet. Results and discussion: After evaluating the application of material, it was possible to perceive it as aggregating material for interdisciplinar knowledge in a multidisciplinar team.
\end{abstract}

Keywords: Palliative care; Cancer patient; Quality of life; Educational and promotional materials; Continuing education.

\section{Resumen}

Actualmente, observamos un aumento en la cronicidad de las enfermedades no transmisibles, elevando la estimación de la vida de la población y mostrando la existencia del processo patológico que conduce a la finitude de la vida. Automáticamente surge la necesidad de estabelecer un proceso de asistencia y educación a los Cuidados Paliativos, que simboliza la mejora del sistema Muerte y Agonizante. Objetivo: Sabiendo que la información constitute un 
elemento fundamental en el processo de desarrollo de la Educación Permanente en Salud para el trabajo en la atención al paciente, se retrata la expeiencia en la construcción de un folleto educativo sobre Cuidados Paliativos en un hospital de emergencia de la ciudad de Rio de Janeiro, un vector de conocimiento. Metodología: Se realizo una investigación descriptiva sobre la necesidad de conocimiento de los profesionales de enfermería en el tema de Cuidados Paliativos, que se convirtió en el detonante para la construcción de este folleto. Resultados y discusión: Luego de evaluar la aplicación del material, fue posible percibirlo como material de agregación para el conocimiento interdisciplinario en un equipo multidisciplinario.

Palabras clave: Cuidados paliativos; Paciente con cancer; Calidad de vida; Materiales educativos y de divulgación; Educación permanente.

\section{Introdução}

O envelhecimento populacional nas últimas décadas tem sido evidenciado, e com isso observa-se o aumento da prevalência de câncer e outras doenças não transmissíveis. A isso também pode estar correlacionado ao avanço tecnológico que permite terapêuticas mais desenvolvidas a transformar doenças antes mortais a estado de crônicas, aumentando a longevidade de seus portadores. Apesar de todos os esforços de pesquisadores e cientistas, a busca da cura e/ou maior, melhor, preservação da vida para a qual nós os profissionais de saúde somos treinados, persistem desafios e tabus a serem desvendados, em particular, aqueles relacionados à morte. E infelizmente pode-se observar o aumento de pacientes em quadro 'fora de possibilidades terapêuticas', acumulando-se nos hospitais e recebendo uma assistência inadequada, e provavelmente focada na tentativa de recuperação, onde são utilizados métodos invasivos e alta tecnologia sem sucesso, Academia Nacional de Cuidados Paliativos (2012).

Os cuidados paliativos não dizem respeito somente a cuidados institucionais, trata-se de toda uma filosofia de cuidados que pode e deve ser utilizada em diferentes contextos e outras instituições, que não da saúde. Esta pode ocorrer em domicílio da pessoa portadora de doença não transmissível ou em fase terminal, próxima à finitude da vida, na instituição de saúde onde está internada ou em uma unidade específica de uma instituição de saúde destinada para este fim (Pessini \& Bertachini, 2004).

No Caderno de Atenção Domiciliar do Ministério da Saúde existe a recomendação de que os cuidados paliativos devem ser oferecidos o mais cedo possível no curso de qualquer doença crônica potencialmente fatal. E ainda nesta sugestão reforça aos cuidados paliativos devendo garantir uma abordagem na qual melhora a qualidade de vida dos pacientes e suas famílias, na presença de problemas associados a doenças que ameacem a vida, mediante prevenção e alívio de sofrimento pela detecção precoce e tratamento de dor ou outros problemas físicos, psicológicos, sociais e espirituais, estendendo inclusive a fase do luto (Lei No 12.732, De 22 De Novembro De 2012, 2012).

A educação torna-se também primordial quando o assunto abordado são os cuidados paliativos, e Maciel et al. (2016) os trata de forma ímpar quando ressalta que se faz necessária a existência de diferentes níveis de educação para as diversas profissões da área de saúde, de acordo com as necessidades de cada uma e a especificidade de cada profissão. Ainda aborda se que o nível de educação necessário deve ser ajustado ao desenvolvimento do cuidado paliativo na prática diária.

Conforme Ceccim (2005) ressalta a Educação Permanente em Saúde se configura em uma mudança na formação profissional dos agentes de saúde, em se tratando do brasileiro podemos observar diversos movimentos de mudança na atenção em saúde facilitando a melhoria na qualidade profissional. Esta qualidade fica evidencia-se quando fornecidos conteúdos, instrumentos e recursos à formação técnica, humanística, e submetidos a um projeto de mudanças. Faz-se ímpar usar a multiplicidade de vivências profissionais aliada a projetos integrados ao setor de trabalho e aos mundos do ensino.

Seguindo a linha da Educação Permanente na Saúde podemos afirmar que materiais educativos são dispositivos facilitadores do processo ensino-aprendizagem, que permite a transferência de conhecimento mediante envolvimento e a participação do indivíduo e possibilitando a troca de experiências adequadas ao aprimoramento de habilidades inclusive profissionais. Com isto estes materiais têm sido largamente utilizados para educação em saúde, na qual pode representar um 
veículo de socialização do conhecimento (Salci et al, 2013), e serem produzidos no decorrer dos processos de trabalho das equipes profissionais, multiprofissionais e interprofissionais (Batista, 2012).

Este artigo tem o objetivo de apresentar a construção de uma cartilha educativa a respeito de Cuidados Paliativos em hospital de emergência, de forma a ser um vetor de estímulo ao conhecimento.

\section{Metodologia}

Trata-se de um estudo qualitativo descritivo, do tipo relato de experiência. Obteve-se como cenário um grande hospital de emergência, referência em trauma-ortopedia, da capital fluminense no Brasil.

A construção da cartilha faz parte do estudo de dissertação de mestrado da autora, que após a realização de um teste piloto onde foram realizadas 10 entrevistas com a equipe de enfermagem de uma clínica cirúrgica. Este grupo de profissionais foi composto por quatro (04) enfermeiros e seis (06) técnicos e auxiliares de enfermagem.

Após este piloto foi constatado a necessidade de um maior esclarecimento acerca do assunto, pois foi observado que $80 \%$ da amostra possuía conhecimento parcial ou nenhum sobre cuidados paliativos, demonstrando o baixo grau de conhecimento. Setenta por cento dos voluntários ainda possuíam a errônea ideia de que cuidados paliativos é o mesmo que deixar morrer, ou seja, o não-cuidado. Foi também observado que todos os participantes deste projeto piloto tinham a necessidade em saber se as respostas fornecidas na entrevista estavam corretas, e alguns solicitaram referências de material científicos para obterem mais conhecimento a respeito.

Em virtude desta constatação procedeu-se a elaboração de um projeto onde pudesse ser suprimida a maioria das dúvidas e aguçasse o conhecimento acerca de cuidados paliativos.

Conhecendo as dificuldades enfrentadas pelos profissionais da enfermagem quanto a disponibilidade de tempo, optouse em elaborar uma cartilha impressa onde pudesse ser ofertada após as entrevistas e levassem consigo, tendo a oportunidade de ler em momento mais adequado e confortável facilitando a ampliação de seu conhecimento. Após a leitura da cartilha foi solicitado aos participantes que respondesse de forma voluntária a um questionário avaliando e sugerindo observações caso sentisse necessidade.

Iniciou-se a produção a partir da pesquisa de conteúdo científicos para embasamento das orientações a serem fornecidas aos profissionais. Foram utilizados referenciais científicos básicos sobre a temática como manuais da ANCP (2012), orientações de conselho regional profissional, tratado legal e livros de autores especialistas no assunto, conforme citados nas referências deste artigo.

Tendo recolhido este material iniciou-se a construção da cartilha sempre evocando que a linguagem deveria ser acessível a todos, pois deveria servir como facilitador da compreensão do conhecimento sobre cuidados paliativos, independentemente do nível de escolaridade e social de quem fosse utilizá-lo. A elaboração procurou ter como um dos objetivos estimular, e até mesmo tendo a pretensão convidar ao leitor a trabalhar com a filosofia paliativista. As ilustrações foram realizadas por um artista voluntário que trabalha no próprio hospital como maqueiro, que ao saber da construção da cartilha sentiu necessidade de contribuir com o trabalho realizado.

Quando a cartilha ficou com o conteúdo elaborado, considerado minimamente suficiente, foi enviado a alguns profissionais pontuais para avaliação do material e posterior contribuições caso sentissem necessidade. Enviada ao responsável pela educação continuada do hospital, a médica responsável pela oncologia na secretaria de saúde do município do Rio de Janeiro e para o vice-presidente da Academia Nacional de Cuidados Paliativos no estado do Rio de Janeiro, na perspectiva de articulações intra e interinstitucionais. Todos estes profissionais após a leitura da cartilha aprovaram e puderam ceder suas contribuições com sugestões. Com estas sugestões foi encaminhada para impressão em gráfica própria onde o custo ficou sob a responsabilidade integral da mestranda. Com essa versão do produto pronto, foi o momento de iniciar a coleta de dados da 
pesquisa e finalizar com a distribuição da cartilha, tendo como ponto ápice o convite à leitura e o preenchimento do questionário avaliando a cartilha. Inicialmente, mesmo antes de analisar as respostas da avaliação pude observar a aceitação positiva do material, tendo recebido o convite para levar a cartilha a outro serviço onde se pratica a paliação no estado do Rio de Janeiro, e sua região metropolitana.

Materiais educativos avaliados por instrumentos de conteúdo na área da saúde devem visar facilitação do trabalho dos profissionais de saúde para orientação e educação de pacientes e familiares (Áfio, 2014). Acrescenta-se que devem possuir por finalidade promover a saúde, garantindo efetividade do cuidado para a população-alvo. Logo, o domínio "objetivo" de um instrumento é necessário para o entendimento acerca do conteúdo a ser estudado.

\section{Resultados}

A construção do material educativo, conforme Leite et al (2018) acrescenta como agregador de conteúdo aos profissionais da saúde, teve início a partir dos conteúdos selecionados através das maiores dúvidas a respeito do tema, sendo realizada a busca nos referenciais teóricos indicados na discussão com o grupo participante da pesquisa. Desenvolveu-se um material-instrumento educativo intitulado provisoriamente: "Cartilha", contendo 16 páginas.

Pode-se afirmar que os textos da cartilha são predominantemente em voz ativa, de forma que transpareça uma conversa com o leitor. Buscou-se formar frases curtas e uma linguagem popular de forma a facilitar a compreensão de todos em consonância com as afirmações feitas por Rocha et al. (2019).

Descritivo do material

A cartilha foi organizada de forma que a capa foi construída com a representação nas laterais acima dos brasões da prefeitura do Rio de Janeiro e do Mestrado Profissional de Ensino na Saúde (MPES) da UFF. Logo abaixo uma representação artística de acolhimento feito pelo artista Renato, onde conseguiu traduzir através do desenho um elemento ímpar da filosofia paliativista e finalizando uma citação de Saunders (2018).

Na primeira página foi inserida uma citação de Krübler-Ross (2012), que foi uma psiquiatra pioneira no tratamento de pacientes até então vistos como terminais nos EUA, foi uma pioneira junto com Saunders (2018) e outros incentivadores do modelo hospice de tratamento. Durante toda construção da cartilha foi inserido citações de personalidades da filosofia paliativista.

Na página subsequente foi realizado agradecimentos a especialistas que muito contribuíram na construção do material.

Na sequência o sumário, apresentação, agradecimentos aos profissionais que contribuíram, o conteúdo e referências bibliográficas

Na Figura 1, abaixo, apresenta-se a diagramação da cartilha com seus elementos principais em sua construção base. 
Figura 1: Diagramação da Cartilha.

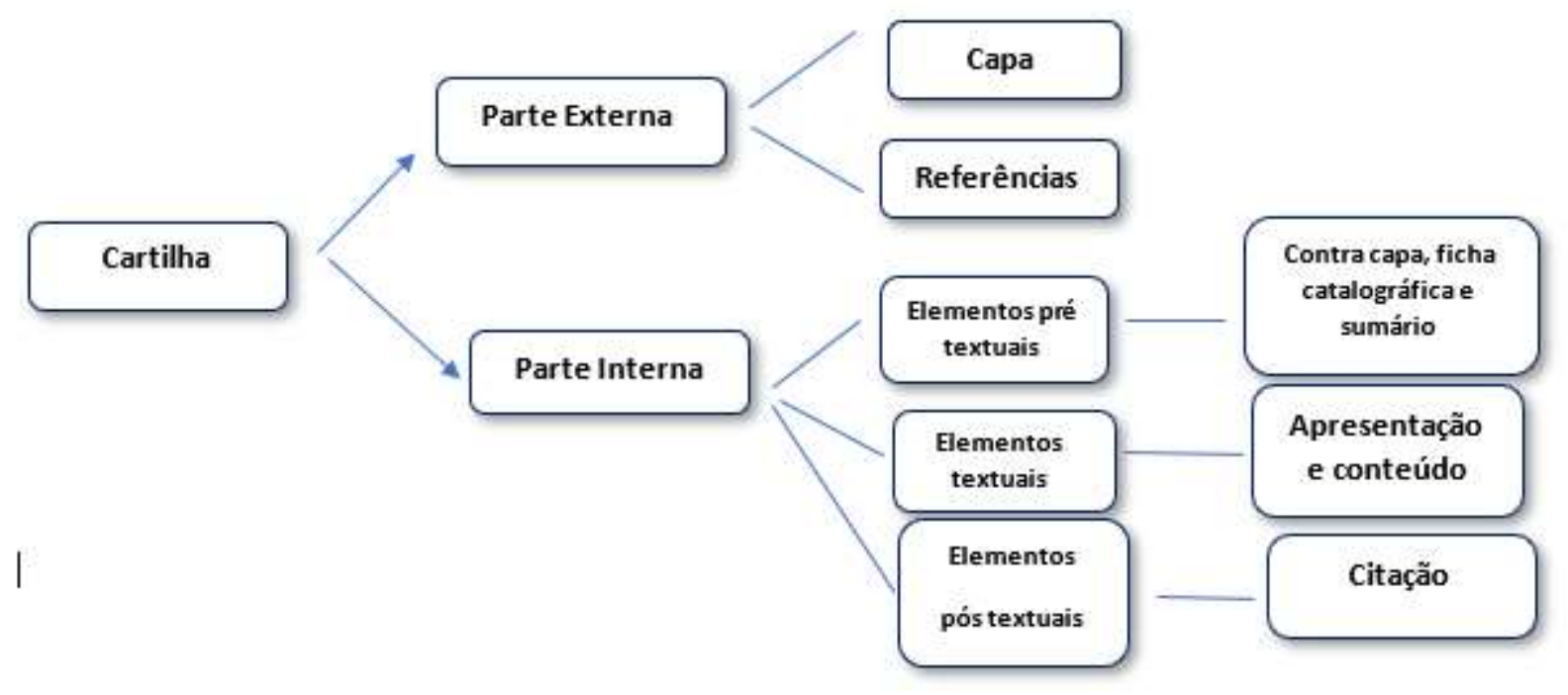

Fonte: Diagramação representativa da cartilha organizada pela autora (2021).

\section{Discussão}

A Cartilha foi idealizada por uma problematização encontrada durante o piloto realizado no desenvolvimento do mestrado da autora, sendo assim observou-se a relevância da construção desta para o impacto na qualidade da assistência prestada aos pacientes com indicação de paliação. A aplicação prévia do instrumento na pequena amostra, caracterizada como piloto, permitiu a construção de escolhas metodológicas mais adequadas. Reafirmou-se a necessidade do desenvolvimento de uma tecnologia educacional que fosse acessível e de fácil compreensão aos profissionais foco e quiçá posteriormente aos usuários.

Em Silvestre et al (2012) e Ceccim (2004) podemos encontrar evidências que justificam o uso de cartilhas educativas como material que auxilia a mudanças significativas no aprendizado, auxiliando na contribuição de conhecimentos.

Em confluência com alguns autores como: Lima et al (2017) e Barros, Pizarro e Lopes Junior (2013), a utilização de cartilha como uma tecnologia educativa tem demonstrado grande aceitação pelos profissionais e pela comunidade em geral, e existem diversos estudos a respeito do desenvolvimento desta tecnologia em diferentes temáticas. Sendo os resultados obtidos a partir desta tecnologia extremamente satisfatórios e com isto despertando de forma vertiginosa o interesse de pesquisadores na intenção do aperfeiçoamento desta tecnologia.

$\mathrm{Na}$ área de saúde os materiais educativos devem visar a facilitação do trabalho dos profissionais para a orientação e educação tanto dos pacientes quanto aos profissionais. Estes materiais devem possuir a finalidade de promover a saúde garantindo a efetividade do cuidado, ou seja, deve garantir o foco como instrumento necessário para o entendimento acerca do conteúdo a ser estudado (Áfio, 2014).

Faz-se importante manter à coesão e coerência sobre o conteúdo e que se mantenha o foco na temática proposta, tendo os seus parágrafos, ou trechos em sequência lógica de ideias, onde se parte de questões gerais e impessoais para assuntos delicados e específicos. Sendo a suficiência um dos aspectos estreitamente relacionados à veracidade dos materiais, sendo que conteúdos educativos explanam tópicos relevantes da temática proposta de forma abrangente, evitando dúvidas ao leitor. (Filatro, 2011)

Pode-se afirmar que o uso da cartilha disfarça o propósito em ser persuasiva em confluência com as afirmações de Schineider et al. (2020) de modo que representa uma alternativa positiva para atingir a um público que pode ter um acesso restrito a informação, seja em decorrência da escolarização, a falta de familiaridade com o assunto, ou pela falta de letramento 
que envolva a leitura de artigos, boletins médicos, periódicos. Por estes possíveis motivos acredita-se que pelo perfil dos participantes deste estudo, esta tecnologia tem grande potencial para contribuir no incremento no conhecimento do públicoalvo.

\section{Conclusão}

Conclui-se que a cartilha desenvolvida é um material educativo elaborado de forma participativa com grande potencial de contribuir ao conhecimento dos profissionais não só da enfermagem, mas a todos que frequentem as dependências do hospital confirmando o empoderamento citado por Oliveira (2014).

Considerando que esta cartilha representa um recurso ao fortalecimento do conhecimento profissional e agregador das relações profissionais pacientes, à medida que possui a capacidade dos materiais educativos em promover a melhoria na qualidade da assistência prestada.

Os materiais educativos podem ser fontes de educação e adaptados a linguagem de domínio do público-alvo, podendo ser facilmente compreensíveis, objetivos e claros, promovendo através da informação a redução do medo, ansiedade e facilitando a adesão na filosofia paliativista.

A construção desta cartilha conseguiu atingir o objetivo de sensibilizar aos profissionais quanto a temática delicada como a abordagem da finitude da vida, desbravando tabus e até criando um chamamento dos profissionais para a discussão. Acredita-se que a cartilha se constituiu uma tecnologia ilustrada capaz de favorecer o diálogo entre os profissionais para o reconhecimento dos pacientes com indicação a paliação estimulando ao questionamento dos cuidados oferecidos ao paciente oncológico internado, com vistas a propiciar o conforto e promover qualidade de vida ao paciente oncológico em finitude de vida.

Através da construção desta cartilha podemos ter a contribuição para continuidade de instrumento atualizado na continuidade do trabalho de educação permanente na saúde em cuidados paliativos no mesmo hospital e em outros serviços.

\section{Referências}

Academia Nacional de Cuidados Paliativos. (2012). Manual de Cuidados Paliativos ANCP Ampliado e atualizado (2ºd). ANCP.

Áfio, A. C. E., Balbino, A. C., Alves, M. D. S., Carvalho, L. V. de., Santos, M. C. L., \& Oliveira, N. R. (2014). Analysis of the concept of nursing educational technology applied to the patient. Revista da Rede de Enfermagem do Nordeste, 15(1), 158-165. https://doi.org/10.15253/2175-6783.2014000100020.

Barros, B. C. S. N., Pizarro, M. V., \& Lopes Junior, J. (2013). Atas do IX Encontro Nacional de Pesquisa em Educação em Ciências, IX ENPE. A caracterização do uso de narrativas quadrinizadas nos livros didáticos de Ciências do PNLD 2013 no contexto dos anos iniciais do Ensino Fundamental.

Batista, N. A. (2012). Educação Interprofissional em Saúde: Concepções e Práticas. Caderno FNEPAS, 2. http://www.fnepas.org.br/artigos_caderno/v2/educacao_interprofissional.pdf.

Ceccim, R. B. (2005). Educação Permanente em Saúde: Descentralização e disseminação de capacidade pedagógica na saúde. Ciência \& Saúde Coletiva, 10(4), 975-986. https://doi.org/10.1590/S1413-81232005000400020

Ceccim, R. B., \& Feuerwerker, L. C. M. (2004). O quadrilátero da formação para a área da saúde: Ensino, gestão, atenção e controle social. Physis: Revista de Saúde Coletiva, 14(1), 41-65.

Filatro A (2011). Design instrucional contextualizado: educação e tecnologia ( $3^{\mathrm{a}}$ ed.). SENAC.

Kübler-Ross, E. (2012). Sobrea morte e o morrer: o que os doentes terminais têm para ensinar a médicos, enfermeiras e aos seus próprios parentes. WMF Martins Fontes.

Lei N $N^{\circ}$ 12.732, De 22 De Novembro De 2012, no 12.732 (2012). http://www.planalto.gov.br/ccivil_03/_ato2011-2014/2012/lei/112732.htm

Leite, S. de S., Áfio, A. C. E., Carvalho, L. V. de, Silva, J. M. da., Almeida, P. C. de., \& Pagliuca, L. M. F. (2018). Construction and validation of an Educational Content Validation Instrument in Health. Revista Brasileira de Enfermagem, 71(suppl 4), 1635-1641. https://doi.org/10.1590/0034-7167-20170648 .

Lima, A. C. M. A. C. C., Bezerra, K. de C., Sousa, D. M. do N., Rocha, J. de F., \& Oriá, M. O. B. (2017). Construção e Validação de cartilha para prevenção da transmissão vertical do HIV. Acta Paulista de Enfermagem, 30(2), 181-189. https://doi.org/10.1590/1982-0194201700028. 
Research, Society and Development, v. 10, n. 7, e57810716926, 2021

(CC BY 4.0) | ISSN 2525-3409 | DOI: http://dx.doi.org/10.33448/rsd-v10i7.16926

Oliveira, S. C. de., Lopes, M. V. de O., \& Fernandes, A. F. C. (2014). Development and validation of an educational booklet for healthy eating during pregnancy. Revista Latino-Americana de Enfermagem, 22(4), 611-620. https://doi.org/10.1590/0104-1169.3313.2459.

Vicens, M. do C., et al (Org.). (2016). Enfermagem em cuidados paliativos. Letra Editorial. http://www.corensc.gov.br/wp-content/uploads/2016/11/CuidadosPaliativos-Parte-1-Site.pdf

Pessini, L., \& Bertachini, L. (2006). Humanização e cuidados paliativos. (3ª ed.). Edições Loyola, p. 181-204.

Rocha, E., Paes, R., Sthal, G., \& Souza, A. (2019). Cuidados Paliativos: Cartilha educativa para cuidadores de pacientes oncológicos. Clinical \& Biomedical Research, 39(1). https://seer.ufrgs.br/hcpa/article/view/85741

Salci, M. A., Maceno, P., Rozza, S. G., Silva, D. M. G. V. da., Boehs, A. E., \& Heidemann, I. T. S. B. (2013). Health education and its theoretical perspectives: A few reflections. Texto \& Contexto - Enfermagem, 22(1), 224-230. https://doi.org/10.1590/S0104-07072013000100027.

Saunders, C. (2011). 'The last frontier: first published in cicely saunders: selected writings. Oxford Scholarship Online, p. 180-186.

Saunders, C. (2018). Velai Comigo - Inspiração para uma vida em Cuidados Paliativos. (1 $1^{\mathrm{a}}$ ed.).

Schneider, L. G., Heinz, M. K., Kwiatkowiski, H. S., Kolhs, M., \& Silva, D. T. de R. e. (2020). Construção de cartilha informativa sobre a COVID-19: Relato de experiência PET- Saúde/ Interprofissionalidade. Saúde e meio ambiente: revista interdisciplinar, 9(Supl.1), 37-38. https://doi.org/10.24302/sma.v9iSupl.1.3413

Silvestre, L. J. B., Mota, F. R. L., Ribeiro, J., \& Costa, I. (2012). A aplicação das novas tecnologias de informação e comunicação no âmbito da práxis de Enfermagem. Anais do XIII Congresso Brasileiro em Informática em Saúde - CBIS 2012. XIII Congresso Brasileiro em Informática em Saúde - CBIS 2012. https://silo.tips/queue/a-aplicaao-das-novas-tecnologias-de-informaao-e-comunicaao-no-ambito-da-praxis-d?\&queue_id=-

$1 \& v=1623885299 \& u=M T c 3 L j E 5 M i 40 L j g 0$. 